\title{
Ulinastatin attenuates lipopolysaccharide-induced cardiac dysfunction by inhibiting inflammation and regulating autophagy
}

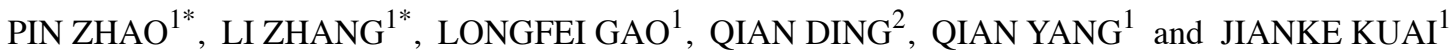 \\ ${ }^{1}$ Department of Anesthesiology, Third Hospital of Xi'an, Xi'an, Shaanxi 710018; ${ }^{2}$ Department of Anesthesiology, \\ Tangdu Hospital, The Second Affiliated Hospital of Air Force Medical University, Xi'an, Shanxi 710038, P.R. China
}

Received August 23, 2019; Accepted March 27, 2020

DOI: $10.3892 /$ etm.2020.8755

\begin{abstract}
Ulinastatin exerts protective effects against lipopolysaccharide (LPS)-induced cardiac dysfunction. Autophagy has been demonstrated to serve an important role in sepsis-induced cardiomyopathy; however, whether ulinastatin has an anti-autophagic effect in sepsis requires further investigation. The present study aimed to determine the protective effects of ulinastatin on cardiac dysfunction and its role in autophagy during sepsis. $\mathrm{C} 57 \mathrm{BL} / 6 \mathrm{~J}$ mice were randomly divided into a control, LPS and LPS + ulinastatin group, the survival status of the mice was observed every $6 \mathrm{~h}$ and the survival rate at each time point was calculated for 7 days. Furthermore, JC-1 dye and ELISAs were used to analyze the mitochondrial membrane potential (MMP) and serum cardiac troponin I (cTnI) levels, respectively. Western blotting and ELISAs were used to measure the levels of tumor necrosis factor (TNF)- $\alpha$ and interleukin (IL)-6. In addition, the cardiac ultrastructure and the number of autophagosomes formed were visualized using transmission electron microscopy, and the pathological changes in the myocardial tissues were analyzed using hematoxylin \& eosin staining. Finally, the expression levels of autophagy-related proteins were analyzed using western blotting and immunofluorescence staining. The current study indicated that ulinastatin significantly improved the survival rate of septic mice. It was suggested that ulinastatin may protect against LPS-induced myocardium injury through its anti-inflammatory activity, as decreased cTnI levels, increased MMP and decreased expression levels of TNF- $\alpha$ and IL- 6 were all observed following ulinastatin treatment. Furthermore, the number of autophagosomes formed, and the expression levels of microtubule-associated
\end{abstract}

Correspondence to: Professor Jianke Kuai or Professor Qian Yang, Department of Anesthesiology, Third Hospital of Xi'an, 10 Fengcheng Three Road, Xi'an, Shaanxi 710018, P.R. China E-mail: 15129875749@163.com

E-mail: 454979818@qq.com

*Contributed equally

Key words: ulinastatin, autophagy, sepsis, cardiomyopathy protein light chain 3 and Beclin 1 were significantly decreased following ulinastatin treatment. It was further observed that ulinastatin suppressed LPS-induced autophagosome formation, as indicated by the accumulation of sequestosome $1 / \mathrm{p} 62$, and the elimination of lysosome-associated membrane glycoprotein 1 . In conclusion, the results of the present study suggested that ulinastatin treatment may improve survival and exert a protective effect over LPS-induced cardiac dysfunction. Furthermore, this protective effect may be associated with its anti-inflammatory and anti-autophagic activity.

\section{Introduction}

Sepsis is a systematic inflammatory response and it is a major cause of mortality in intensive care units (1). Moreover, sepsis is associated with the dysfunction of multiple organs (1). It has been previously documented that $40-50 \%$ of patients with sepsis develop cardiac dysfunction (2). Cardiac dysfunction is a major feature of sepsis, with the $50 \%$ mortality rate increasing in patients with severe heart failure $(3,4)$. The mortality rate of sepsis is rising despite the increasing numbers of studies developing antimicrobial therapies and circulatory support (5). Therefore, the present study aimed to determine the mechanisms of LPS-induced myocardial injury to facilitate the development of novel treatment strategies.

Ulinastatin is an endogenous inhibitor of proteases that is located in the urine and blood, and inhibits various serine protease (6). Ulinastatin has been previously used to treat sepsis cardiomyopathy, due to its ability to function as a protease inhibitor and its anti-inflammatory effects (7). It was previously demonstrated that ulinastatin enhanced cardiac function, reduced the size of the myocardial infarction and decreased the levels of inflammatory cytokines during hemorrhagic shock and ischemia-reperfusion (IR) injury (8). A previous study has also suggested that the protective effects of ulinastatin are based on its anti-inflammatory, anti-oxidative stress and anti-apoptotic properties (9). Furthermore, ulinastatin was indicated to serve an important role in treating lipopolysaccharide (LPS)-induced myocardial injury (10). However, the specific mechanisms of the role of ulinastatin in sepsis are not fully understood.

Autophagy is a type of programmed cell death and it is an adaptation used to respond to a variety of stress stimuli (11). Previous studies have identified the presence of activated 
autophagy in a variety of heart diseases, such as IR injury, heart failure and sepsis $(12,13)$. It has been widely demonstrated that autophagy performs two opposing functions in the heart (14); it serves a protective role during nutrient deprivation and cellular stress, whereas the excessive induction of autophagy promotes self-destruction (15). In a model of IR injury, ulinastatin was revealed to protect cardiomyocytes via the activation of the mTOR pathway, which is associated with the process of autophagy (16). A previous study has identified that ulinastatin exerted a protective effect on sepsis through its regulation of autophagy in myocardial IR injury models (16). Therefore, it was hypothesized that ulinastatin may serve a protective role in sepsis-induced myocardial injury by regulating autophagy; however, further research into the role of ulinastatin in sepsis is required. The present study aimed to investigate whether ulinastatin protected against sepsis cardiomyopathy and to determine the possible underlying mechanisms of this interaction.

\section{Materials and methods}

Sepsis model. All procedures were approved by The Animal Ethics Committees of The Fourth Military Medical University (Xi'an, China) and were performed in accordance with the guidelines of The China Council of Animal Care. A total of 84 C57BL/6J mice (age, 8-10 weeks; weight, 25-30 g; sex, male) were purchased from The Laboratory Animal Center of The Fourth Military Medical University. Mice were housed at a temperature of $25^{\circ} \mathrm{C}$ in $\sim 60 \%$ humidity, with a $12 \mathrm{~h}$ light/dark cycle. Mice were provided with a standard diet and free access to water. Endotoxemia was induced via an intraperitoneal (i.p.) injection of $18 \mathrm{mg} / \mathrm{kg}$ lipopolysaccharide (LPS; cat. no. L2630; Sigma-Aldrich; Merck KGaA) or $10 \mathrm{mg} / \mathrm{kg}$ LPS from the Escherichia coli serotype O111:B4 (17). Ulinastatin was obtained from Guangdong Tianpu Biochemical Pharmaceutical Co., Ltd. To determine the protective effect of ulinastatin on the survival rate following lethal endotoxemia, 60 mice were divided into two groups: i) A total of 30 mice in the LPS group, where mice were treated with the lethal dose of $18 \mathrm{mg} / \mathrm{kg}$ LPS and $0.9 \%$ saline; and ii) 30 mice in the LPS + Ulinastatin group, where the mice received $18 \mathrm{mg} / \mathrm{kg}$ LPS and i.p. injection of $1 \times 10^{5} \mathrm{U} / \mathrm{kg}$ Ulinastatin (i.p.) daily for 4 days, which was determined in a previous study (17).

To investigate the effects of ulinastatin on cardiac function and the levels of autophagy, 24 mice were randomly divided into a control group, a LPS group (mice received $10 \mathrm{mg} / \mathrm{kg} \mathrm{LPS}$ and $0.9 \%$ saline) and a LPS + ulinastatin group [mice received $10 \mathrm{mg} / \mathrm{kg}$ LPS and $1 \times 10^{5} \mathrm{U} / \mathrm{kg}$ Ulinastatin (i.p.)] (17), with 8 mice in each group. The administered dose of ulinastatin was selected according to a previous study, in which ulinastatin was observed to exhibit a protective effect on sepsis (18). All mice were anesthetized for echocardiography after treated with LPS and/or Ulinastatin for $12 \mathrm{~h}$, following which they were sacrificed for subsequent experiments.

For survival analysis, humane endpoints were established. In the process of observing the survival of mice, once they showed labored breathing, they were euthanized immediately by i.p. injection of $120 \mathrm{mg} / \mathrm{kg}$ sodium pentobarbital sodium $(20 \mathrm{mg} / \mathrm{ml})$. Conventional anti-shock therapy was given by an intraperitoneal injection of $0.9 \%$ saline after 4 days of drug treatment. At the end of the 7 day survival cycle, the rest of the surviving mice in two groups were euthanized using $120 \mathrm{mg} / \mathrm{kg}$ sodium pentobarbital sodium $(20 \mathrm{mg} / \mathrm{ml})$ through the intraperitoneal route. Following cervical dislocation to ensure death, $600 \mu \mathrm{l}$ blood samples were collected from the abdominal aorta and the myocardial tissues of mice were collected and frozen at $80^{\circ} \mathrm{C}$ for further evaluation. To minimize animal suffering, only qualified personnel were permitted to perform the experiments.

Echocardiography. After anesthesia with an i.p. injection of $60 \mathrm{mg} / \mathrm{kg}$ sodium pentobarbital sodium $(20 \mathrm{mg} / \mathrm{ml})$, conventional echocardiography of the left ventricle (LV) in each mouse was performed $12 \mathrm{~h}$ after an i.p. injection of LPS using a mouse echocardiography system (Vevo 2100 Imaging System; VisualSonics, Inc.) that was equipped with a $30-\mathrm{MHz}$ phased transducer. The following parameters were measured: LV end diastolic pressure (LVEDP), LV developed pressure (LVDP), maximal velocity increase of LV pressure per second (+dP/dtmax) and maximal velocity decrease of LV pressure per second (-dP/dtmax).

ELISAs. Blood samples were centrifuged at 1,500 $\mathrm{x} \mathrm{g}$ for $15 \mathrm{~min}$ at room temperature to collect the serum. Serum cardiac troponin-I (cTnI; cat. no. F00503; Shanghai Westang Bio-Tech Co., Ltd.), cardiac tumor necrosis factor (TNF)- $\alpha$ (cat. no. T7539; Sigma-Aldrich; Merck KGaA) and cardiac interleukin (IL)-6 (cat. no. 555240; BD Biosciences) levels were analyzed using their respective ELISA kits, according to the manufacturer's protocol.

Mitochondrial membrane potential (MMP). A mitochondria isolation kit (cat. no. C3606; Beyotime Institute of Biotechnology) was used to extract the purified mitochondria from the myocardial tissues, according to the manufacturer's protocol (19). Subsequently, a mitochondrial membrane potential assay kit with JC-1 dye (cat. no. C2006; Beyotime Institute of Biotechnology) and a fluorescence microplate reader (Chromate 4300; Awareness Technology, Inc.) were used to measure MMP, according to the manufacturer's protocol. JC-1 green fluorescence $(530 \mathrm{~nm})$ was used to reflect JC-1 monomers and red fluorescence $(590 \mathrm{~nm})$ was used to determine the formation of J-aggregates. The ratio of J-aggregates to JC-1 mitochondria were measured using a microplate reader after JC-1 staining. The ratio of monomeric to aggregated JC-1 fluorescence intensity was used to quantify the changes in MMP. Data were presented as the normalized percentage of the average fluorescence intensity and values were normalized to the control group.

Western blotting. Total proteins were extracted using the RIPA protein extraction reagent (Beyotime Institute of Biotechnology) from the myocardial tissues and the protein concentration was determined using the bicinchoninic acid Protein Assay kit (Beyotime Institute of Biotechnology). A total of $20 \mu \mathrm{g}$ protein/lane was separated via 10-15\% SDS-PAGE. The separated proteins were subsequently transferred onto PVDF membranes (EMD Millipore) and blocked with 5\% non-fat milk at room temperature for $1 \mathrm{~h}$. The membranes were then incubated with the following primary antibodies overnight at 
$4^{\circ} \mathrm{C}$ : Anti-TNF- $\alpha$ (1:1,000; cat. no. ab6671; Abcam), anti-IL-6 (1:1,000; cat. no. ab9324; Abcam), anti-microtubule-associated protein light chain 3 LC3B (1:1,000; cat. no. ab51520; Abcam), anti-lysosomal-associated membrane protein 1 (LAMP-1; 1:1,000; cat. no. ab24170; Abcam), anti-sequestosome-1 (SQSTM1)/p62 (1:1,000; cat. no. ab56416; Abcam) and anti-GAPDH (1:3,000; cat. no. ab181602; Abcam). Following the primary antibody incubation, the membranes were washed three times with TBS- $0.1 \%$ Tween 20 and incubated with horseradish peroxidase-conjugated secondary antibody (1:5,000; cat. no. ZB-2301; Beijing Zhongshan Golden Bridge Biotechnology Co., Ltd.) and horseradish peroxidase-conjugated secondary antibody (1:5,000; cat. nos. ab6728; Abcam) for $2 \mathrm{~h}$ at room temperature. Protein bands were visualized using an enhanced chemiluminescence kit (EMD Millipore) and expression levels were quantified using a densitometric analysis system (Image Lab software; version 4.0; Bio-Rad Laboratories, Inc.).

Hematoxylin \& eosin $(H \& E)$ staining. Following the establishment of the LPS model, myocardial tissues were excised, washed with ice-cold PBS, following which 1-mm thick slices were prepared after the hearts were snap frozen at $-80^{\circ} \mathrm{C}$ for $5 \mathrm{~min}$. The slices were fixed directly in $10 \%$ neutral formalin for $24 \mathrm{~h}$ at room temperature and were subsequently paraffin-embedded. Sections ( $3 \mu \mathrm{m}$ thickness) were then prepared from the paraffin-embedded tissue blocks by dehydration through a graded ethanol series and washing with xylene for H\&E staining, for $12 \mathrm{~min}$ at room temperature. Morphological changes in the myocardial tissues were evaluated using an Olympus light microscope (magnification x400; Olympus Corporation).

Transmission electron microscopy (TEM). Myocardial tissues were sliced into $1 \mathrm{~mm}^{3}$ sections and fixed with $2.5 \%$ glutaraldehyde overnight at $4^{\circ} \mathrm{C}$. The tissues were immersed in $1 \%$ osmium tetroxide for $2 \mathrm{~h}$ at room temperature, dehydrated in an ascending ethanol series, followed by dehydration in an $100 \%$ acetone for three times at $4^{\circ} \mathrm{C}$ and subsequently embedded in epoxy resin. The tissues were then cut into ultrathin sections (60-70 nm) using an ultramicrotome. Sections were stained with $2 \%$ uranyl acetate and $2 \%$ lead citrate for $30 \mathrm{~min}$ at room temperature. Stained sections were visualized using a JEM-1010 TE microscope (magnification x15,000 and $\mathrm{x} 30,000$; JEOL, Ltd.) and the amount of autophagosome were quantified using Gatan Digital Micrograph ${ }^{\circledR}$ software (Gatan, Inc.).

Immunofluorescence staining. Myocardial tissues were excised, washed with ice-cold PBS, following which 1-mm thick slices were prepared after the hearts were snap frozen at $-80^{\circ} \mathrm{C}$ for $5 \mathrm{~min}$. The slices were sequentially fixed with $4 \%$ formaldehyde for $30 \mathrm{~min}$ at room temperature, permeabilized with $0.2 \%$ Triton X-100 (HyClone; GE Healthcare Life Sciences) for $15 \mathrm{~min}$ at room temperature and blocked in 5\% bovine serum albumin (Beyotime Institute of Biotechnology) for $1 \mathrm{~h}$ at room temperature. Sections were then incubated with a rabbit anti-LC3 antibody (1:100; cat. no. ab128025; Abcam) and a rabbit anti-Beclin 1 antibody (1:100; cat. no. ab210498; Abcam) at $4^{\circ} \mathrm{C}$ overnight. Following the primary antibody

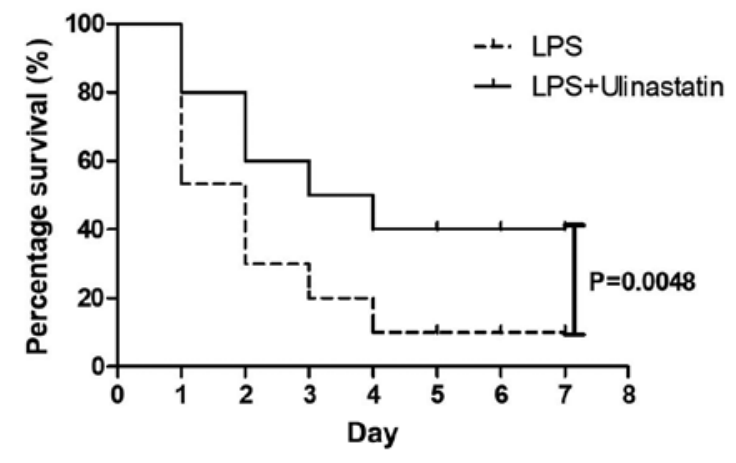

Figure 1. Ulinastatin improves the survival rate of mice with endotoxemia The survival rate of mice receiving a lethal dose of LPS $(18 \mathrm{mg} / \mathrm{kg})$ or LPS $(18 \mathrm{mg} / \mathrm{kg})+$ ulinastatin $\left(1 \times 10^{5} \mathrm{U} / \mathrm{kg}\right)$ was analyzed for 7 consecutive days LPS, lipopolysaccharide.

incubation, the sections were incubated with an Alexa Fluor ${ }^{\circledR}$ 488-conjugated goat anti-rabbit IgG secondary antibody (1:10; cat. no. ab150077; Abcam) at $37^{\circ} \mathrm{C}$ for $1 \mathrm{~h}$. The cell nucleus was stained with $2 \mu \mathrm{g} / \mathrm{ml}$ DAPI for $30 \mathrm{~min}$ at room temperature and $500 \mathrm{ml} / \mathrm{l}$ glycerine was used to seal the sections. The slides were observed using a confocal laser scanning microscope (magnification $x 400$ ) to analyze the expression levels of autophagy-associated proteins.

Statistical analysis. All experiments were independently performed in triplicate. Statistical analysis was performed using SPSS version 19 software (IBM Corp.) and data are presented as the mean \pm SEM. Survival rate analysis was analyzed using the Kaplan-Meier method, with a log-rank test used for comparison (20). Statistical differences between groups were determined using a one-way ANOVA, followed by a Student-Newman-Keuls test for multiple comparisons. $\mathrm{P}<0.05$ was considered to indicate a statistically significant difference.

\section{Results}

Ulinastatin improves the survival rate of mice with endotoxemia. To determine the protective effect of ulinastatin on the survival rate following lethal endotoxemia, mice were treated with a lethal dose of LPS $(18 \mathrm{mg} / \mathrm{kg})$, whereas mice in the LPS + ulinastatin group received $18 \mathrm{mg} / \mathrm{kg}$ LPS and ulinastatin $\left(1 \times 10^{5} \mathrm{U} / \mathrm{kg}\right)$ daily for 4 days daily. After 7 days, it was revealed that only $12 / 30$ of mice in the LPS + ulinastatin group survived, thus the survival rate was $40 \%$ (Fig. 1). In the LPS group, mice were treated with an equal amount of saline and it was subsequently demonstrated that only $3 / 30$ mice had survived 7 days later, thus demonstrating a survival rate of $10 \%$ (Fig. 1). Therefore, these findings suggested that ulinastatin may reduce the mortality rate of lethal endotoxemia.

Effect of ulinastatin on the cardiac function in LPS-stimulated mice. The cardiac function parameters in each experimental group are presented in Table I. Following the establishment of the LPS model mice, it was revealed that the LVEDP was significantly increased compare with that in the control group. The LVDP, $+\mathrm{dP} / \mathrm{dtmax}$ and $-\mathrm{dP} / \mathrm{dtmax}$ were all found to be significantly decreased following $12 \mathrm{~h}$ of treatment in the LPS 
Table I. Effect of ulinastatin on cardiac function in LPS-induced mice.

\begin{tabular}{|c|c|c|c|c|c|}
\hline Group & $\mathrm{n}$ & $\begin{array}{l}\text { Left ventricle } \\
\text { end diastolic } \\
\text { pressure (mmHg) }\end{array}$ & $\begin{array}{l}\text { Left ventricle } \\
\text { developed } \\
\text { pressure (mmHg) }\end{array}$ & $\begin{array}{l}\text { Maximal velocity } \\
\text { increase of left } \\
\text { ventricle pressure } \\
\text { per second (mmHg) }\end{array}$ & $\begin{array}{l}\text { Maximal velocity } \\
\text { decrease of left } \\
\text { ventricle pressure } \\
\text { per second (mmHg) }\end{array}$ \\
\hline Control & 8 & $20.7 \pm 3.6$ & $118.1 \pm 11.7$ & $8,720.6 \pm 135.1$ & $6,121.0 \pm 212.0$ \\
\hline LPS & 8 & $42.1 \pm 1.8^{\mathrm{a}}$ & $68.2 \pm 7.3^{\mathrm{a}}$ & $6,012.4 \pm 312.4^{\mathrm{a}}$ & $3,132.4 \pm 254.7^{\mathrm{a}}$ \\
\hline LPS + ulinastatin & 8 & $36.5 \pm 1.3^{\mathrm{a}, \mathrm{b}}$ & $89.0 \pm 5.6^{\mathrm{a}, \mathrm{b}}$ & $6,956.2 \pm 216.3^{\mathrm{a}, \mathrm{b}}$ & $4,528.1 \pm 198.5^{\mathrm{a}, \mathrm{b}}$ \\
\hline
\end{tabular}

${ }^{\mathrm{a}} \mathrm{P}<0.05$ vs. control group; ${ }^{\mathrm{b}} \mathrm{P}<0.05$ vs. LPS group. LPS, lipopolysaccharide.

group compared with the control group (Table I). Moreover, following ulinastatin treatment, the LVEDP was significantly decreased by $13.3 \%$, whereas the LVDP, $+\mathrm{dP} / \mathrm{dtmax}$ and $-\mathrm{dP} / \mathrm{dtmax}$ were increased by $30.5,15.7$ and $44.6 \%$, respectively, compared with the LPS group.

Effects of ulinastatin on the mice myocardium during endotoxemia. The tissues and serum from individual mice were obtained following $12 \mathrm{~h}$ of LPS induction. The ELISA results revealed that cTnI levels were significantly increased in the LPS and LPS + ulinastatin groups compared with the control group (Table II). Of note, the LPS + ulinastatin group had significantly decreased cTnI levels compared with the LPS group (Table II). It was also indicated that the MMP was significantly decreased in the LPS and LPS + ulinastatin groups compared with the control group; however, the LPS + ulinastatin group demonstrated a significantly increased MMP compared with the LPS group (Table II). Previous studies have reported that inflammatory cytokines serve an important role in the progression of sepsis (21). To investigate the protective effects of ulinastatin on the LPS-induced inflammatory response, the levels of TNF- $\alpha$ and IL- 6 in the mice myocardial tissue were also investigated. The ELISA results indicated that the levels of TNF- $\alpha$ and IL-6 were significantly increased in the LPS and LPS + ulinastatin groups compared with the control group (Table II). However, following the treatment with ulinastatin, the levels of TNF- $\alpha$ and IL- 6 were significantly decreased in the myocardium of mice with endotoxemia compared with the LPS group (Table II).

Effects of ulinastatin on the expression levels of inflammatory factors in endotoxemia. The western blotting data revealed that the protein expression levels of TNF- $\alpha$ and IL- 6 were significantly increased in the LPS and LPS + ulinastatin groups compared with the control group (Fig. 2A and B). Notably, following treatment with ulinastatin, the expression levels of TNF- $\alpha$ and IL- 6 were significantly decreased in the myocardium of mice with endotoxemia in the LPS + ulinastatin group compared with those in the LPS group (Fig. 2A and B). Collectively, these findings suggested that ulinastatin may exert protective effects in the endotoxemia myocardium and may alleviate the inflammatory response through decreasing the expression levels of TNF- $\alpha$ and IL- 6 .

Effects of ulinastatin on the cardiac ultrastructure and pathological changes. TEM was used to observe the cardiac ultrastructure; a normal cardiac structure and mitochondrial distribution were observed in the control group (Fig. 3A), whereas in the LPS group, the myofilaments and mitochondria were disorganized in the myocardial tissue, alongside the presence of autophagy (Fig. 3B). Notably, in the LPS + ulinastatin group, the myocardial tissue exhibited orderly arranged myofilaments, a normal mitochondrial distribution and the presence of small magnitudes of autophagy (Fig. 3C).

H\&E staining was subsequently used to assess the pathological changes in the myocardial tissue. In the LPS group, myofibril loss, myocardial cell necrosis and structural abnormalities were all observed, which indicated the occurrence of severe cardiac injury (Fig. 3E). However, in the LPS + ulinastatin group, there was a reduction in the pathological damage observed in the mice hearts compared with the LPS group (Fig. 3F).

Ulinastatin decreases the levels of LPS-induced autophagy. Myocardial tissues from the mice were obtained $12 \mathrm{~h}$ after LPS induction. It was subsequently demonstrated that the expression levels of LC3II were significantly increased in the LPS and LPS + ulinastatin group compared with the control group (Fig. 4A). However, the expression levels of LC3II (Fig. 4A), Beclin 1 (Fig. 4B) and LAMP-1 (Fig. 4C) were all significantly decreased following ulinastatin treatment compared with those in the LPS group. In addition, it was revealed that the expression levels of SQSTM1/p62 was significantly decreased in the LPS and LPS + ulinastatin group compared with those in the control group, ulinastatin significantly increased the expression levels of SQSTM1/p62 further compared with those in the LPS group (Fig. 4D). Moreover, TEM was also used to observe the number of autophagosomes formed in the heart of each group. A normal mitochondrial structure and very few autophagosomes were observed in the control group, whereas in the LPS group, myocardial tissues exhibited a significantly increased number of autophagosomes compared with the control group. Notably, ulinastatin treatment was observed to significantly decrease this LPS-induced increase in autophagosome formation (Fig. 4E).

Furthermore, the expression levels of the autophagy associated proteins, LC3 and Beclin 1, were analyzed using confocal microscopy. The expression levels of LC3 and Beclin 1 in the control group were low, whereas under 
Table II. Effect of ulinastatin on the mouse myocardium during endotoxemia.

\begin{tabular}{lccccc}
\hline Group & n & $\begin{array}{c}\text { Mitochondrial } \\
\text { membrane potential }\end{array}$ & $\begin{array}{c}\text { Serum cardiac } \\
\text { troponin I (ng/ml) }\end{array}$ & $\begin{array}{c}\text { Tumor necrosis } \\
\text { factor- } \alpha(\mathrm{pg} / \mathrm{ml})\end{array}$ & Interleukin-6 (pg/ml) \\
\hline Control & 8 & 1.00 & $0.136 \pm 0.0142$ & $39.620 \pm 2.140$ & $3.820 \pm 0.430$ \\
LPS & 8 & $0.321 \pm 0.067^{\mathrm{a}}$ & $0.919 \pm 0.0138^{\mathrm{a}}$ & $268.420 \pm 15.920^{\mathrm{a}}$ & $14.560 \pm 0.920^{\mathrm{a}}$ \\
LPS + ulinastatin & 8 & $0.673 \pm 0.075^{\mathrm{a}, \mathrm{b}}$ & $0.585 \pm 0.0129^{\mathrm{a}, \mathrm{b}}$ & $172.370 \pm 8.560^{\mathrm{a}, \mathrm{b}}$ & $9.270 \pm 0.610^{\mathrm{a}, \mathrm{b}}$ \\
\hline
\end{tabular}

${ }^{\mathrm{a}} \mathrm{P}<0.05$ vs. control group; ${ }^{\mathrm{b}}<0.05$ vs. LPS group. LPS, lipopolysaccharide.

A
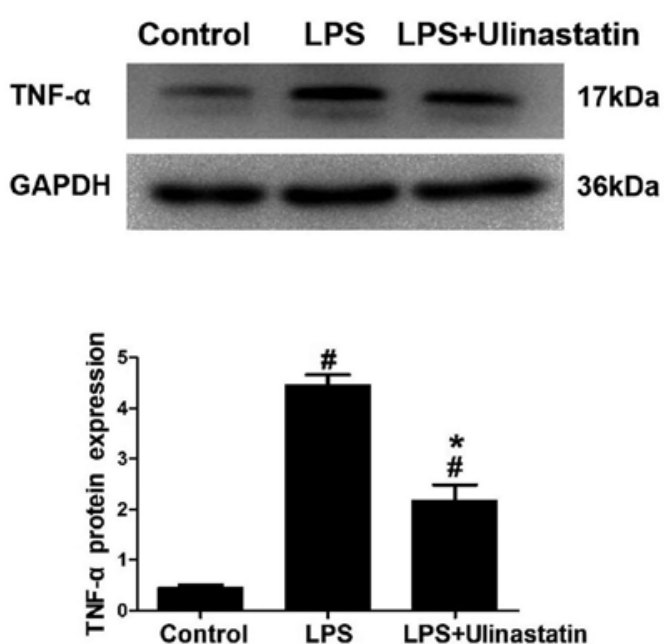

B

\section{Control LPS LPS+Ulinastatin}

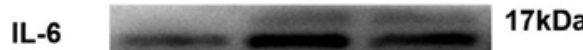

GAPDH $36 \mathrm{kDa}$

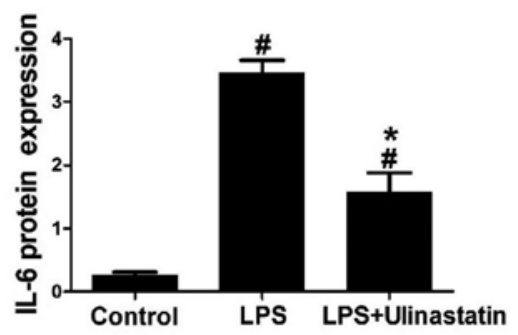

Figure 2. Effect of ulinastatin on the expression levels of inflammatory factors in endotoxemia. Western blotting was used to analyze the protein expression levels of (A) TNF- $\alpha$ and (B) IL-6 in myocardial tissues. GAPDH was used as the loading control. Data are presented as the mean \pm SEM and statistical differences were determined using a one-way ANOVA and Student-Newman-Keuls post hoc test. ${ }^{\prime \prime} \mathrm{P}<0.05$ vs. control group; " $\mathrm{P}<0.05$ vs. LPS group. TNF- $\alpha$, tumor necrosis factor- $\alpha$; IL-6, interleukin-6; LPS, lipopolysaccharide.
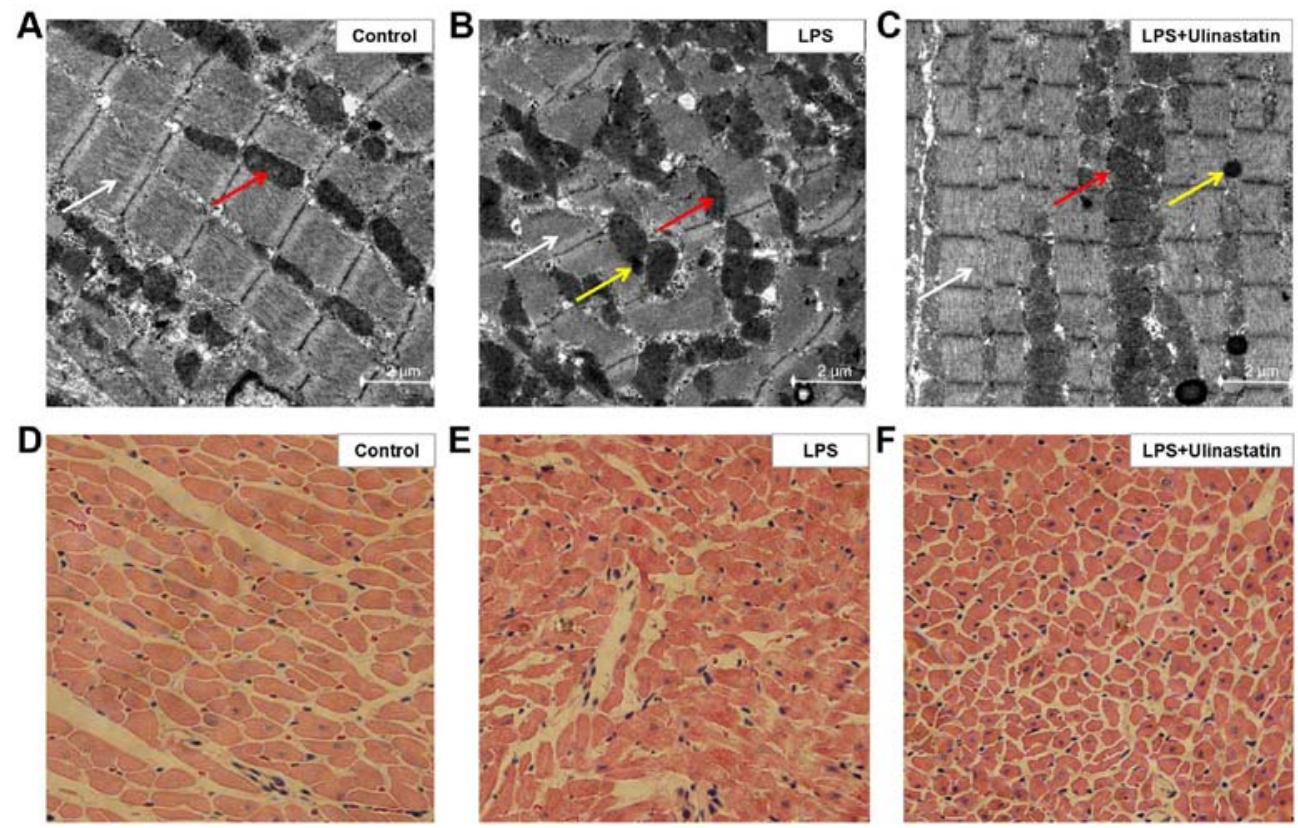

Figure 3. Effect of ulinastatin on attenuating myocardial injuries. LPS-induced endotoxemia mice were treated with saline or ulinastatin, and the left ventricle tissues were collected $12 \mathrm{~h}$ later for analysis. Transmission electron microscopy was used to analyze the cardiac ultrastructure in the (A) control, (B) LPS and (C) LPS + ulinastatin groups. Magnification x15,000. Scale bar, $2 \mu \mathrm{m}$. White arrow indicates the myofilaments, the red arrow indicates the mitochondria and the yellow arrow indicates the presence of autophagosomes. Hematoxylin \& eosin staining was used to determine the pathological changes in the myocardial tissue in the (D) control, (E) LPS and (F) LPS + ulinastatin groups. Magnification x200. LPS, lipopolysaccharide. 
A
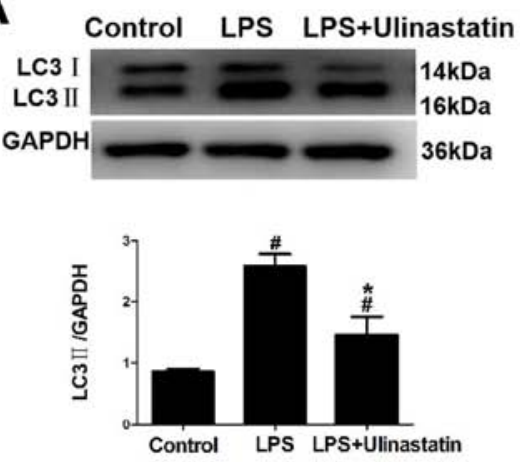

C
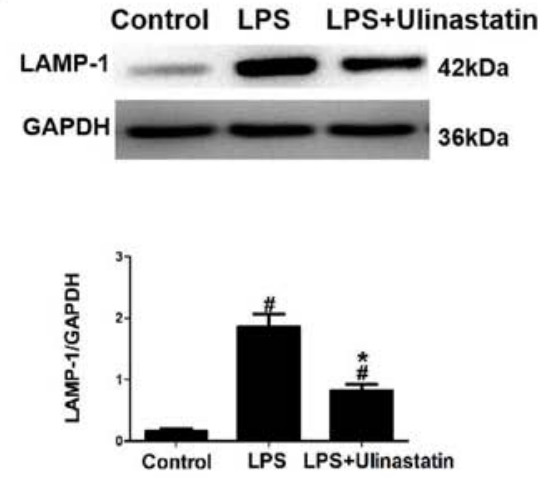

B
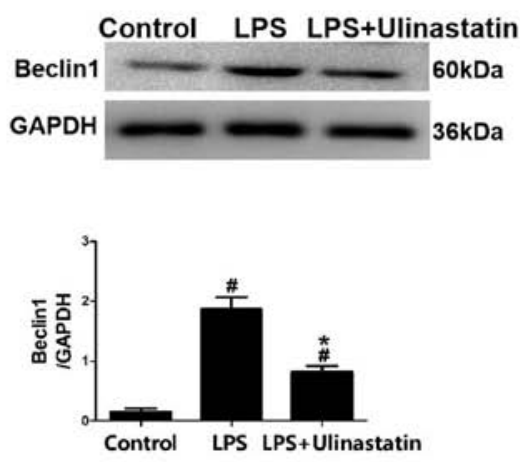

D

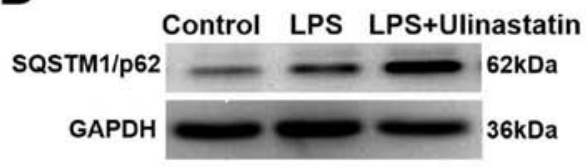

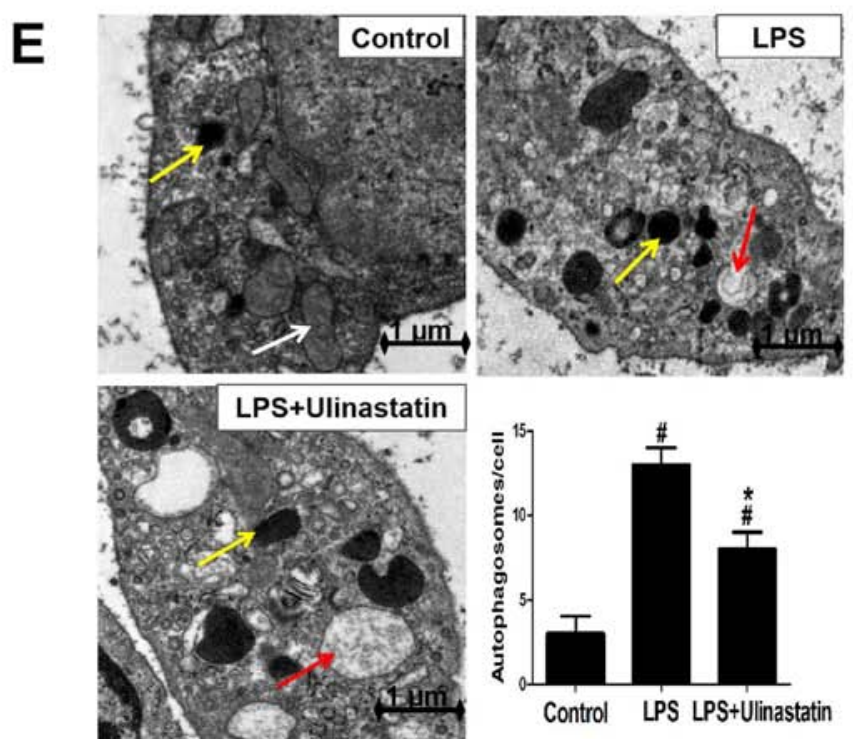

Figure 4. Effects of ulinastatin on autophagy in mice with endotoxemia. LPS-induced endotoxemia mice were treated with saline or ulinastatin, and the left ventricle tissues were collected $12 \mathrm{~h}$ later for analysis. Western blotting was used to analyze the protein expression levels of (A) LC3II, (B) Beclin 1, (C) LAMP-1 and (D) SQSTM1/p62 in the myocardial tissues. GAPDH was used as a loading control. (E) Transmission electron microscopy was used to observe the number of autophagosomes formed in the cardiac tissue. Magnification x30,000. Scale bar, $1 \mu \mathrm{m}$. Yellow arrow indicates the autophagosomes, the white arrow indicates the mitochondria and the red arrow indicates the lysosomes. Data are presented as the mean \pm SEM. Data were compared using a one-way ANOVA, followed by a Student-Newman-Keuls post hoc test. " $\mathrm{P}<0.05$ vs. control group; ${ }^{*} \mathrm{P}<0.05$ vs. LPS group. LPS, lipopolysaccharide; LC3, microtubule-associated protein light chain 3; LAMP, lysosomal-associated membrane protein 1; SQSTM1, sequestosome-1.

the same conditions, the fluorescent intensities of LC3 and Beclin 1 in the LPS group were significantly increased compared with the control group (Fig. 5). Notably, following the treatment with ulinastatin, the expression levels of LC3 and Beclin 1 were significantly decreased compared with the LPS group (Fig. 5).

\section{Discussion}

The present study demonstrated that ulinastatin may improve survival rate and exert a protective effect against LPS-induced cardiac dysfunction. It was suggested from the findings of the present study that this protective effect may be associated with 


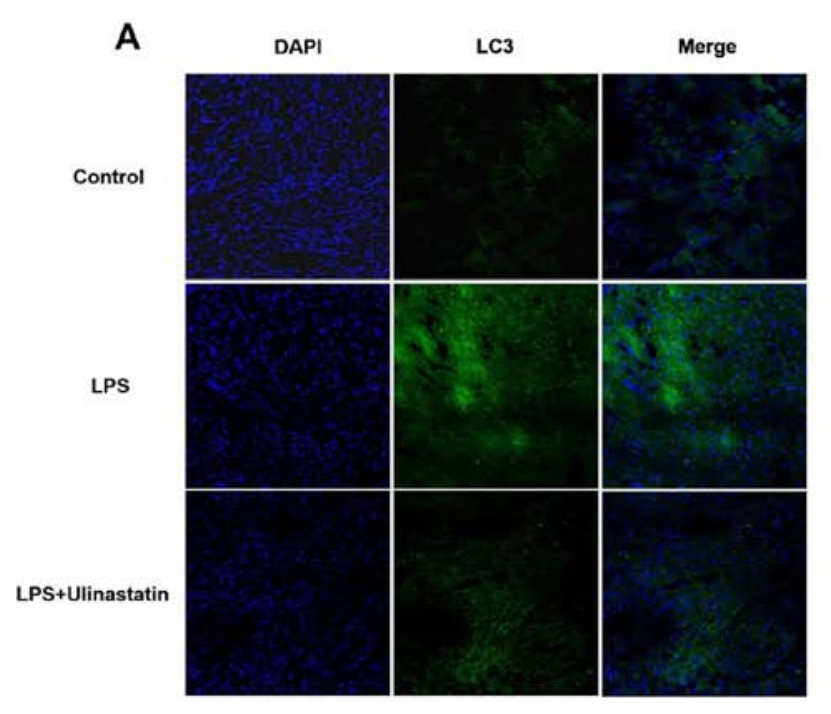

C

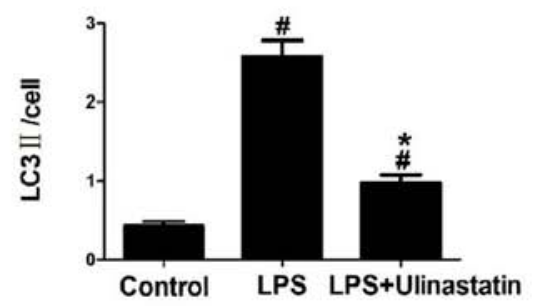

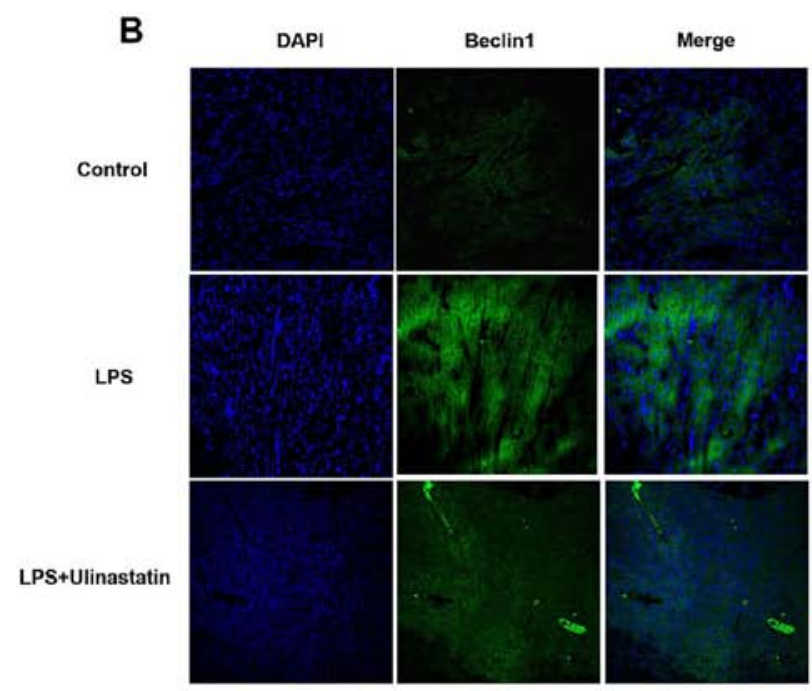

D

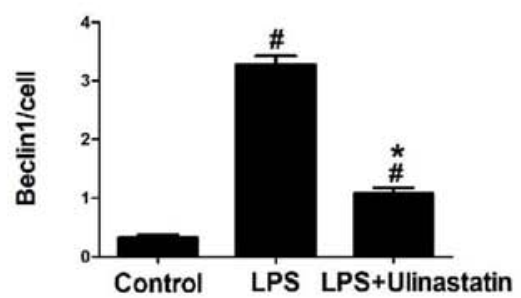

Figure 5. Effect of ulinastatin on autophagy in endotoxemia mice. Endotoxemia mice were treated with vehicle or ulinastatin, and the left ventricle tissues were collected $12 \mathrm{~h}$ later for analysis. A confocal microscope was used to analysis the fluorescence distribution of (A) LC3II and (B) Beclin 1 in myocardial tissues. Semi-quantitative analysis of (C) LC3II and (D) Beclin 1 expression levels in myocardial tissues. Magnification x200. Data were analyzed using a one-way ANOVA, followed by a Student-Newman-Keuls post hoc test. ${ }^{~} \mathrm{P}<0.05$ vs. control group; ${ }^{*} \mathrm{P}<0.05$ vs. LPS group. LPS, lipopolysaccharide; LC3, microtubule-associated protein light chain 3.

the anti-inflammatory activity of ulinastatin and its ability to inhibit autophagy. Therefore, the administration of ulinastatin may exhibit a protective effect in the pathophysiology of sepsis.

A previous study has reported the presence of decreased serum levels of ulinastatin in patients with sepsis, and the levels of ulinastatin were at their lowest during cases of severe sepsis and septic shock (22). Therefore, treatment with ulinastatin may increase the levels of ulinastatin in patients with severe sepsis. The protective effect of ulinastatin in sepsis has been confirmed in a number of previous studies; for example, ulinastatin was observed to serve a protective role in the heart tissue of septic rats, and its mechanism was attributed to its regulatory effect over the stress response, cell signaling transduction, energy metabolism, the immune response and other related genes $(23,24)$. Furthermore, a previous study revealed that ulinastatin contributed to the recovery of cardiac function following reperfusion by reducing mitochondrial dysfunction and maintaining energy production (8). However, the role that ulinastatin serves over the regulation of autophagy during sepsis remains poorly understood. Therefore, in the present study, endotoxemic mice were treated with ulinastatin to investigate its underlying mechanism.

TNF- $\alpha$ and IL- 6 are cytokines associated with the inflammatory response (25). A previous study indicated that ulinastatin reduced sepsis-related inflammation by downregulating TNF- $\alpha$ expression levels (26). ELISA and western blotting data from the present study revealed that the serum levels and protein expression levels of TNF- $\alpha$ and IL- 6 were significantly increased in endotoxemia mice, and these LPS-induced increases could be significantly decreased following ulinastatin treatment. Therefore, the results of the present study indicated that ulinastatin may protect against LPS-induced myocardial injury by inhibiting the release of TNF- $\alpha$ and IL- 6 .

Ulinastatin has been proposed to serve as a myocardial protective agent, and it has been reported that ulinastatin improved the myocardial contractility, reduced the myocardial infarct size and decreased the levels of creatine kinase and cTnI in myocardial IR injury (16). cTnI is a highly sensitive and specific marker of myocardial injury (27). Mitochondria are important organelles for cardiomyocytes that not only provide the energy for cells, but can also trigger cell apoptosis (28). Furthermore, decreases in the MMP have been found to be the driving force for mitochondrial dysfunction (29). In the present study, endotoxemic mice were treated with ulinastatin and it was indicated that ulinastatin exhibited a protective effect in the hearts of mice with endotoxemia. This protective effect was demonstrated by decreased cTnI levels, an increased MMP, as well as the presence of an intact, well-ordered cardiac ultrastructure.

Autophagy serves an essential role in cell survival, as well as in cell death (14). Autophagy is a highly regulated intracellular degradation process, by which cells remove cytosolic long-lived proteins and damaged organelles (15). LC3 is the mammalian homologue of yeast Atg8, and as a lipidated form of LC3, LC3II has been widely used as a marker of autophagy 
by indicating the number of autophagosomes formed (30). Moreover, Beclin 1 has been discovered to serve an important role in autophagosome formation and autolysosomal fusion (31), and LAMP-1 is a lysosome marker that has been associated with the increased accumulation of autophagic vacuoles (32). Furthermore, p62 is a marker of autophagic flux and impaired autophagy is often accompanied by p62 accumulation, which results in large p62/ubiquitinated protein aggregates (33). Previous studies have demonstrated that the LPS-induced autophagic flux and autophagy were at their highest levels $12 \mathrm{~h}$ after LPS intraperitoneal injection (34). Therefore, the present study chose to investigate the effect of ulinastatin at this time point. Western blotting and TEM data obtained from the present study revealed that the expression levels of LC3II and the number of autophagosomes formed were significantly decreased following ulinastatin treatment. Furthermore, it was revealed that ulinastatin reduced the LPS-induced autophagosome maturation, which was demonstrated through the decreased protein expression levels of LAMP-1 and the increased expression levels of SQSTM1/p62. Therefore, the present study results suggested that the decreased activation of autophagy may be a critical protective mechanism of ulinastatin in mice with endotoxemia.

In conclusion, the findings of the present study suggested that ulinastatin may serve a cardioprotective role in sepsis, which may be achieved through its ability to suppress inflammation and autophagy. These findings may provide a clinical basis for the use of ulinastatin as a novel therapeutic option for the treatment of sepsis-related cardiac dysfunction. However, there are several limitations to the present study. Whilst the present study was able to observe changes in autophagy, there was a lack of experiments using specific autophagy inhibitors 3-methyladenine or autophagy agonists such as rapamycin. Therefore, future studies should further investigate the effects of ulinastatin on autophagy-related pathways. Additionally, the present study did not investigate the effect of ulinastatin at multiple time points or in in vitro cell models. As the present experimental set-up may have also produced bias in the results, further extensive research is required before solid conclusions can be made.

\section{Acknowledgements}

Not applicable.

\section{Funding}

The present study was funded by the Foundation of Shanxi Provincial [grant no. 2018SF-095(JK.K)].

\section{Availability of materials and methods}

The datasets used and/or analyzed during the present study are available from the corresponding author on reasonable request.

\section{Authors' contributions}

PZ and JKK designed the study. PZ, LZ, LFG, QD and QY performed the experiments. Data were collated by LZ and the results of data were discussed by LFG and QY. PZ prepared the figures. $\mathrm{PZ}$ and $\mathrm{LZ}$ wrote the first draft of the manuscript. All authors read and approved the final version of the manuscript.

\section{Ethics approval and consent to participate}

All procedures were approved by The Animal Ethics Committees of The Fourth Military Medical University (Xi'an, China) and were performed in accordance with the guidelines of The China Council of Animal Care.

\section{Patient consent for publication}

Not applicable.

\section{Competing interests}

The authors declare that they have no competing interests.

\section{References}

1. Hotchkiss RS and Karl IE: The pathophysiology and treatment of sepsis. N Engl J Med 348: 138-150, 2003.

2. Kumar A, Haery C and Parrillo JE: Myocardial dysfunction in septic shock. Crit Care Clin 16: 251-287, 2000.

3. Rudiger A and Singer M: Mechanisms of sepsis-induced cardiac dysfunction. Crit Care Med 35: 1599-1608, 2007.

4. You W, Min X, Zhang X, Qian B, Pang S, Ding Z, Li C, Gao X, Di R, Cheng Y and Liu L: Cardiac-Specific expression of heat shock protein 27 attenuated endotoxin-induced cardiac dysfunction and mortality in mice through a PI3K/Akt-dependent mechanism. Shock 32: 108-117, 2009.

5. Fleischmann C, Scherag A, Adhikari NK, Hartog CS, Tsaganos T, Schlattmann P, Angus DC and Reinhart K; International Forum of Acute Care Trialists: Assessment of global incidence and mortality of hospital-treated sepsis. Am J Respir Crit Care Med 193: 259-272, 2016.

6. Jönsson-Berling BM, Ohlsson $\mathrm{K}$ and Rosengren $\mathrm{M}$ : Radioimmunological quantitation of the urinary trypsin inhibitor in normal blood and urine. Biol Chem Hoppe Seyler 370: 1157-1161, 1989.

7. Shin IW, Jang IS, Lee SM, Park KE, Ok SH, Sohn JT, Lee HK and Chung YK: Myocardial protective effect by ulinastatin via an anti-inflammatory response after regional ischemia/reperfusion injury in an in vivo rat heart model. Korean J Anesthesiol 61: 499-505, 2011.

8. Masuda T, Sato K, Noda C, Ikeda KM, Matsunaga A, Ogura MN, Shimizu K, Nagasawa H, Matsuyama N and Izumi T: Protective effect of urinary trypsin inhibitor on myocardial mitochondria during hemorrhagic shock and reperfusion. Crit Care Med 31: 1987-1992, 2003.

9. Koga Y, Fujita M, Tsuruta R, Koda Y, Nakahara T, Yagi T, Aoki T, Kobayashi C, Izumi T, Kasaoka S, et al: Urinary trypsin inhibitor suppresses excessive superoxide anion radical generation in blood, oxidative stress, early inflammation, and endothelial injury in forebrain ischemia/reperfusion rats. Neurol Res 32: 925-932, 2010.

10. Yu Z, Rayile A, Zhang X, Li Y and Zhao Q: Ulinastatin protects against lipopolysaccharide-induced cardiac microvascular endothelial cell dysfunction via downregulation of lncRNA MALAT1 and EZH2 in sepsis. Int J Mol Med 39: 1269-1276, 2017.

11. Mizushima N: The ATG conjugation systems in autophagy. Curr Opin Cell Biol 31: 1-10, 2019.

12. Cursio R, Colosetti P and Gugenheim J: Autophagy and liver ischemia-reperfusion injury. Biomed Res Int 2015: 417590, 2015.

13. Ho J, Yu J, Wong SH, Zhang L, Liu X, Wong WT, Leung CC, Choi G, Wang MH, Gin T, et al: Autophagy in sepsis: Degradation into exhaustion? Autophagy 12: 1073-1082, 2016

14. Gurusamy N and Das DK: Is autophagy a double-edged sword for the heart? Acta Physiol Hung 96: 267-276, 2009.

15. De Meyer GR and Martinet W: Autophagy in the cardiovascular system. Biochim Biophys Acta 1793: 1485-1495, 2009. 
16. Xiao J, Zhu X, Ji G, Yang Q, Kang B, Zhao J, Yao F, Wu L, Ni X and Wang $Z$ : Ulinastatin protects cardiomyocytes against ischemiareperfusion injury by regulating autophagy through mTOR activation. Mol Med Rep 10: 1949-1953, 2014.

17. Zhao P, Kuai J, Gao J, Sun L, Wang Y and Yao L: Delta opioid receptor agonist attenuates lipopolysaccharide-induced myocardial injury by regulating autophagy. Biochem Biophys Res Commun 492: 140-146, 2017

18. Wang WK, Lu QH, Wang X, Wang B, Wang J, Gong HP, Wang L, $\mathrm{Li} \mathrm{H}$ and Du YM: Ulinastatin attenuates diabetes-induced cardiac dysfunction by the inhibition of inflammation and apoptosis. Exp Ther Med 14: 2497-2504, 2017.

19. Lan R, Zhang Y, Xiang J, Zhang W, Wang GH, Li WW, Xu LL and Cai DF: Xiao-Xu-Ming decoction preserves mitochondrial integrity and reduces apoptosis after $\mathrm{f}$ ocal cerebral ischemiaand reperfusion via the mitochondrial p53 pathway. J Ethnopharmacol 151: 307-316, 2014.

20. Qiu SQ, van Rooijen J, Nienhuis $\mathrm{HH}$, van derVegt B, Timmer-Bosscha H, van Leeuwen-Stok E, Walenkamp AME, van Deurzen CHM, de Bock GH, et al: High hepatocyte growth factor expression in primary tumor predicts better overall survival in male breast cancer. Breast Cancer Res 22: 30, 2020.

21. Lin CM, Chen CR, Wu XQ, Ren JH, Chen SZ, Luo XF, Mei XQ, Shen LY, Guo MX, Ma XD and Yang T: Effects of blood purification on serum levels of inflammatory cytokines and cardiac function in a rat model of sepsis. Blood Purif 44: 40-50, 2017.

22. Lim YP, Bendelja K, Opal SM, Siryaporn E, Hixson DC and Palardy JE: Correlation between mortality and the levels of inter-alpha inhibitors in the plasma of patients with severe sepsis. J Infect Dis 188: 919-926, 2003.

23. Han D, Shang W, Wang G, Sun L, Zhang Y, Wen H and Xu L: Ulinastatin and thymosin $\alpha 1$-based immunomodulatory strategy for sepsis: A meta-analysis. Int Immunopharmacol 29: 377-382, 2015.

24. Wang FY, Fang B, Qiang XH, Yu TO, Zhong JR, Cao J and Zhou LX: The efficacy and immunomodulatory effects of ulinastatinand thymosin $\alpha 1$ for sepsis: A systematic review and meta-analysis. Biomed Res Int 2016: 9508493, 2016.

25. Gogos CA, Drosou E, Bassaris HP and Skoutelis A: Pro-Versus anti-inflammatory cytokine profile in patients with severe sepsis: A marker for prognosis and future therapeutic options. J Infect Dis 181: 176-180, 2000.
26. Chen X, Wang Y, Luo H, Luo Z, Liu L, Xu W, Zhang T, Yang N, Long $X, Z$ hu N, et al: Ulinastatin reduces urinary sepsis-related inflammation by upregulating IL-10 and downregulating TNF- $\alpha$ levels. Mol Med Rep 8: 29-34, 2013.

27. Varvarousis D, Goulas N, Polytarchou K, Psychari SN, Paravolidakis K, Konstantinidou A, Tsoukalas D, Vlad D, Bouki K and Kotsakis A: Biomarkers of myocardial injury and inflammation after permanent pacemaker implantation: The lead fixation type effect. J Atr Fibrillation 10: 1798, 2018.

28. Shires SE and Gustafsson $\AA \mathrm{B}$ : Mitophagy and heart failure. J Mol Med (Berl) 93: 253-262, 2015.

29. Joiner ML, Koval OM, Li JD, He B, Allamargot C, Gao Z, Luczak ED, Hall DD, Fink BD, Chen B, et al: CaMKII determines mitochondrial stress responses in heart. Nature 491: 269-273, 2012.

30. Nishida K, Kyoi S, Yamaguchi O, Sadoshima J and Otsu K: The role of autophagy in the heart. Cell Death Differ 16: 31-38, 2009.

31. Castillo K, Valenzuela V, Matus S, Nassif M, Oñate M, Fuentealba Y, Encina G, Irrazabal T, Parsons G, Court FA, et al: Measurement of autophagy flux in the nervous system in vivo. Cell Death Dis 4: e917, 2013.

32. Eskelinen EL: Roles of LAMP-1 and LAMP-2 in lysosome biogenesis and autophagy. Mol Aspects Med 27: 495-502, 2006.

33. Mathew R, Karp CM, Beaudoin B, Vuong N, Chen G, Chen HY, Bray K, Reddy A, Bhanot G, Gelinas C, et al: Autophagy suppresses tumorigenesis through elimination of p62. Cell 137: 1062-1075, 2009

34. Zhao P, Gao JJ, Jiang J, Peng X, Wu W, Zheng $\mathrm{L}$ and Yao L: Myocardial cells and mitochondrial autophagy in sepsis mice induced by lipopolysaccharide. Xi Bao Yu Fen Zi Mian Yi Xue Za Zhi 32: 177-181, 2016 (In Chinese).

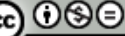

This work is licensed under a Creative Commons Attribution-NonCommercial-NoDerivatives 4.0 International (CC BY-NC-ND 4.0) License. 